\title{
Energieeffizienz in privaten Haushalten im internationalen Vergleich. Eine Policy-Wirkungsanalyse mit QCA
}

\author{
Ines Mayer / Volker Schneider / Claudius Wagemann
}

\begin{abstract}
Energy Efficiency in Private Households in International Comparison. A Policy Impact Analysis with QCA

Abstract: In recent years, the recognition of the multiple benefits related to energy efficiency has become more and more pronounced both at the national and the international level. Therefore, governments worldwide have adopted miscellaneous energy efficiency policies in order to promote the efficient use of energy in all sectors. This study aims at analyzing the impact energy efficiency policies have on the electricity consumption of private households in international comparison. In this context, the study focuses on identifying which policy or combination of policies is most effective, using Qualitative Comparative Analysis (in the fuzzy set version) as a method suited to answer this kind of research question. The results indicate that policies, when applied in combination, are sufficient for the reduction of electricity consumption in private households, with the impact of institutions in combination with norms and standards being the most outstanding. Slow economic growth is identified as an important context factor making it more likely for electricity savings to occur.
\end{abstract}

Keywords: Energy Efficiency, Private Households, Policy Impact Analysis, Qualitative Comparative Analysis (QCA), Two Step Approach

Schlagwörter: Energieeffizienz, private Haushalte, Policy-Wirkungsanalyse, Qualitative Comparative Analysis (QCA), Zwei-Schritt-Ansatz

\section{Einleitung 1}

Weltweit wächst das Bewusstsein für Nachhaltigkeit und die Begrenztheit der natürlichen Ressourcen. Dabei rückt das Thema Energieeffizienz immer stärker ins Blickfeld nationaler und internationaler Diskussionen. Viele Staaten haben sich ehrgeizige Energieeinsparziele gesetzt und die damit verbundenen Anstrengungen zeigen bereits erste Erfolge. Ein breites Spektrum dieser Maßnahmen zielt auf private Haushalte, da in den OECD-Ländern immerhin 30 Prozent des Stroms in privaten Haushalten verbraucht wird, mit steigender Tendenz (IEA 2003, S. 11). Das Einsparpotenzial in diesem Sektor ist nicht unerheblich, es gibt jedoch nur wenig empirisch fundiertes Wissen, mithilfe welcher Instrumente und Mechanismen dieses am besten auszuschöpfen ist. Ziel dieser Studie ist es daher, den Ein-

1 Die Studie wurde im Rahmen des vom BMBF geförderten Projektes „TRANSPOSE“ erstellt. Wir danken ferner den anonymen Gutachtern und der Redaktion der Politischen Vierteljahresschrift für die wertvollen Hinweise und Anregungen sowie Gabi Feistner für das Datenmanagement und die Unterstützung bei der Datenanalyse. 
fluss verschiedener politischer Maßnahmen auf den Stromkonsum und das Einsparverhalten privater Haushalte auf der Basis quantitativer und qualitativer international vergleichbarer Daten zu untersuchen.

Generell ist festzustellen, dass das politikwissenschaftliche Interesse am Thema Energieeffizienz bislang deutlich hinter dem skizzierten Bedeutungszuwachs in der politischen Praxis zurücksteht. Auch für den Bereich der privaten Haushalte sind Analysen zur Wirksamkeit politischer Intervention dünn gesät. Dies gilt insbesondere für empirische Analysen, die über deskriptive Skizzen einzelner Maßnahmen und Politikinstrumente hinausgehen. Für die praktische Politikgestaltung ist aber nicht allein die Frage nach einer effizienten Ausgestaltung einzelner Politiken relevant (siehe dazu Banerjee u. Solomon 2003; Henryson et al. 2000; Nadel 2002; Abrahamse et al. 2005), sondern insbesondere auch das Wissen um deren relative und kombinierte Wirksamkeit. Bislang ist die Frage, wie bestimmte politische Maßnahmen im Vergleich zu anderen wirken, einerseits mit ökonometrischen Methoden (Berry 2008; Yuan et al. 2009), anderseits mit multiplen Fallstudien (Harmelink et al. 2008) untersucht worden. Relativ systematische Übersichten über den Stand der Wirkungsforschung in diesem Politikfeld liefern die Studien von Geller et al. (2006), Gillingham et al. (2006, 2009) und Ürge-Vorsatz et al. (2007). Ein Nachteil der meisten Studien ist, dass entweder nur einzelne Maßnahmen in mehreren Ländern, oder mehrere Maßnahmen in einem Land untersucht werden. Bislang liegen noch keine Studien vor, die ein breites Maßnahmenspektrum in einer hinreichend großen Anzahl von Ländern erfassen, um generalisierungsfähige Aussagen über die differentielle und kombinierte Wirksamkeit öffentlicher Politiken in diesem Politikfeld zu erzeugen.

Unter den Arbeiten, die sich mit der Thematik beschäftigen, nimmt die vorliegende insofern eine Sonderrolle ein, als sie die erste ist, die international vergleichbare Daten über die Wirksamkeit verschiedener Politiken mit der von Charles Ragin entwickelten Qualitative Comparative Analysis (QCA) durchführt. Ein zentraler Vorteil dieser Methode ist es, kausale Schlüsse auch über das Zusammenwirken unterschiedlicher Faktoren im Hinblick auf das Zustandekommen des zu erklärenden Phänomens ziehen zu können, ohne dabei die potentielle Wirkung einzelner Faktoren außer acht lassen zu müssen (Ragin 1987, 2000, 2008). Daher eignet sich QCA besonders zur Beantwortung der Frage nach dem relativen Einfluss verschiedener Politiken, da mit ihr sowohl analysiert werden kann, in welchen Kombinationen verschiedene politische Instrumente zusammenwirken, als auch, welche Instrumente Alternativen zueinander darstellen. QCA wurde als Methode zwar bereits für die Analyse verwandter umweltpolitischer Fragestellungen herangezogen (siehe Befani et al. 2007), neu ist jedoch die Verwendung von QCA im Rahmen der Wirkungsanalyse einer öffentlichen Politik. Wir verwenden dabei die Fuzzy-Set-Variante von QCA (fsQCA) (Ragin 2008), die verschiedene Vorteile für eine Policy-Analyse bietet, wie weiter unten noch näher erläutert wird.

In den folgenden Abschnitten dieser Studie werden wir aus theoretischer Perspektive zunächst fragen, aufgrund welcher Barrieren und Hemmnisse das Potenzial zur Steigerung der Energieeffizienz in privaten Haushalten nicht hinreichend ausgeschöpft wird. Wir beziehen uns hauptsächlich auf politisch-ökonomische Ansätze im weitesten Sinne (für einen Überblick siehe Holzinger 2009). Hieraus 
leiten wir unsere zentralen Hypothesen ab, die sich auf die Wirksamkeit politischer Instrumente im Kontext sozioökonomischer Rahmenbedingungen beziehen. Im dritten Teil geben wir einen Einblick in die methodische Vorgehensweise, um im vierten Teil das Forschungsdesign und die Datenlage zu besprechen. Der fünfte Teil dient der empirischen Analyse, und im abschließenden sechsten Teil werden die Ergebnisse zusammenfassend präsentiert.

\section{Theoretische Einbettung}

Bereits mit den heute zur Verfügung stehenden Technologien wäre es möglich, weitreichende Energieeinsparungen kostendeckend zu erzielen. Nach Schätzungen der Internationalen Energieagentur könnte durch die konsequente Verbreitung der effizientesten Technologien, die heute auf dem Markt erhältlich sind, der Stromverbrauch - bezogen auf den gegenwärtigen Trend - bis zum Jahr 2030 um bis zu 50 Prozent reduziert werden (IEA 2009, S. 22). Obwohl es technisch möglich und wirtschaftlich rentabel wäre, wird das Potenzial zur Einsparung von Energie jedoch nicht einmal annähernd ausgeschöpft (IEA 2007, S. 20). Diese Diskrepanz wird in der Literatur als efficiency gap und als eine Form von Marktversagen bezeichnet (Jaffe u. Stavins 1994).

In der Diskussion um die Gründe für das Auftreten des efficiency gap nimmt die Frage nach den Handlungskalkülen und -motiven privater Verbraucher eine zentrale Rolle ein. Dabei gehen die Sichtweisen weit auseinander. Während die Konsumenten nach der Theorie rationaler Entscheidungen (Rational Choice) ihr Handeln nach rationalen Kriterien ausrichten, hebt die von Herbert Simon (1957) entwickelte Theorie der begrenzten Rationalität hervor, dass das menschliche Wahrnehmungs- und Urteilsvermögen einer Reihe von Beschränkungen unterliege. Die Rational-Choice-Theorie in ,Reinform` ist wenig geeignet, den efficiency gap zu erklären. Wenn energieeffizientes Konsumverhalten wirtschaftlich rentabel ist, dann müsste ein nach Nutzenmaximierung strebender Konsument bemüht sein, die zur Verfügung stehenden Möglichkeiten auszuschöpfen. Allerdings muss berücksichtigt werden, dass Entscheidungen in der Energienutzung nicht immer vollständig durch den Konsumenten bestimmt werden. Ferner können technische, strukturelle und institutionelle Rahmenbedingungen die Entscheidungsspielräume der Konsumenten erheblich einschränken und einer Ausschöpfung der vorhandenen Energieeffizienz-Potenziale im Wege stehen. Ein Beispiel hierzu ist das MieterVermieter- (bzw. Nutzer-Investor-) Dilemma, demzufolge der Vermieter Investitionen in eine energieeffiziente Ausstattung des vermieteten Wohnraums unterlässt, weil nicht er selbst, sondern der Mieter davon profitieren würde (Sorrell 2004, S. 42). Finanzielle Restriktionen und Unsicherheiten wären ein weiterer Grund, warum Verbraucher auch dann nicht in energieeffiziente Produkte oder Technologien investieren, wenn sie diese als langfristig rentabel bewerten (Blumstein et al. 1980, S. 356-357). Schließlich ist Energieeffizienz eine unsichtbare Produkteigenschaft und die Informationsbeschaffung bezüglich des Preis-Leistungsverhältnisses unterschiedlicher Produkte kann daher für den Käufer mit hohen Kosten verbunden sein (Gillingham et al. 2009, S. 606). Wenn aber in der persönlichen Bilanz die Kosten der Informationsbeschaffung den zu erwartenden Nutzen überwiegen, ist 
es unwahrscheinlich, dass Investitionen in Energieeffizienz getätigt, bzw. Anstrengungen unternommen werden, den Energieverbrauch im Haushalt zu senken. Dies gilt umso mehr, als Konsumenten dem Thema Energieeffizienz meist nur wenig Bedeutung beimessen, da Energiekosten nur einen verhältnismäßig geringen Anteil der gesamten Haushaltskosten ausmachen (Brown 2001, S. 1199, 1202).

Selbst unter der Annahme rationalen Verbraucherverhaltens lassen sich also Erklärungen dafür finden, warum die bestehenden Möglichkeiten der Effizienzsteigerung nicht ausreichend genützt werden. Wie bereits erwähnt, gibt es jedoch auch generelle Zweifel an der Gültigkeit der Annahmen des Rational-Choice-Ansatzes. Forschungsergebnisse der Verhaltensökonomie und der kognitiven Psychologie weisen auf das Auftreten kognitiver Anomalien in Entscheidungssituationen hin (einen aktuellen Überblick über diese Forschungsrichtung und deren verbraucherpolitische Relevanz geben Reisch u. Oehler 2009). Beispielsweise werden Entscheidungen oftmals nicht auf der Basis einer bewussten Abwägung von Kosten und Nutzen getroffen, sondern in Übereinstimmung mit Gewohnheiten und einfachen Entscheidungsregeln (Kahneman u. Tversky 2000). Akteure tendieren beispielsweise dazu, bei der Wahl zwischen verschiedenen Alternativen auf diejenige zurückzugreifen, die ihnen vertraut ist (recognition heuristic) (Goldstein u. Gigerenzer 2002). Ein neues, stromsparendes Haushaltsgerät könnte also bei einer Kaufentscheidung von vornherein außer Acht gelassen werden, zugunsten eines ähnlichen, schon bekannten Produkts, das jedoch weniger Stromeinsparung erlaubt.

Kognitive Anomalien treten insbesondere in Entscheidungssituationen auf, die mit Unsicherheiten verbunden sind (McFadden 1999, S. 79). Bei der Wahl zwischen verschiedenen Alternativen werden beispielsweise potenzielle Gewinne und Verluste in Relation zu einem Referenzpunkt, dem Status quo, evaluiert. Der Status quo wird dabei gegenüber anderen Alternativen häufig bevorzugt, da risikoaverse Akteure eher nach einer Minimierung potentieller Verluste streben, als nach Gewinnsteigerung (Kahneman u. Tversky 2000). Investitionen in energieeffiziente Geräte sind daher verglichen mit den zur Verfügung stehenden Alternativen oft nur in begrenztem Maße attraktiv (Brown 2001, S. 1202).

Die unterschiedlichen Konzeptionen menschlichen Verhaltens, wie sie in der Rational-Choice-Theorie respektive dem Bounded-Rationality-Ansatz dargestellt werden, gelten meist als unvereinbare Gegensätze. Demgegenüber gehen wir davon aus, dass beide Sichtweisen ihre Berechtigung haben und einen komplementären Beitrag zum Verständnis des efficiency gap leisten können. Es mag durchaus individuell rational sein, Möglichkeiten zur Steigerung der Energieeffizienz außer Acht zu lassen. Ungünstig gelagerte Rahmenbedingungen mögen der Umsetzung eines Vorhabens, das zu einer verbesserten Energiebilanz führen würde, im Wege stehen. Schließlich mögen auch intrinsische Handlungs- und Wahrnehmungsbarrieren zur suboptimalen Ausnutzung der vorhandenen Potenziale beitragen.

Die Darstellung der hemmenden Faktoren ist nicht nur im Hinblick auf die Erklärung des efficiency gap von Interesse, sondern sie verdeutlicht auch, wo Möglichkeiten bestehen, diese Probleme anzugehen. Beispielsweise wird es den Verbrauchern durch die verpflichtende Einführung von Energieeffizienz-Labeln ermöglicht, informierte Entscheidungen zu treffen, ohne dass dabei Kosten für die 
Informationsbeschaffung anfallen. Ein weiteres Beispiel wären staatliche Beihilfen wie Subventionen oder zinsgünstige Darlehen, welche darauf abzielen, die Anreizstruktur der Konsumenten zu verändern.

Wir ordnen die zur Steigerung der Energieeffizienz zur Verfügung stehenden politischen Maßnahmen nach Art ihrer Wirkungsweise vier Instrumententypen zu und unterscheiden dabei zwischen ökonomischen Anreizen, Informationen, Normen und Standards sowie Institutionen. Diese eingestandenermaßen sparsame Klassifikation orientiert sich an einschlägigen Typologien in der Policy-Forschung und der Steuerungstheorie (Braun u. Giraud 2008; Görlitz u. Burth 1998; Windhoff-Héritier 1987). Jedes dieser Instrumente folgt einer eigenen Logik politischer Steuerung. Während ökonomische Maßnahmen darauf ausgerichtet sind, die Entscheidungsstruktur der Verbraucher durch finanzielle Anreize zu beeinflussen, zielen Informationen darauf ab, die Verbraucher für die Existenz eines Problems zu sensibilisieren, Wissen zu vermitteln und Handlungsoptionen aufzuzeigen. Normen und Standards hingegen bezwecken die mittelbare Beeinflussung des Verbraucherverhaltens über eine Veränderung der Verbraucherumgebung. Mithilfe von Institutionen wie beispielsweise Energieagenturen können unterschiedliche energiepolitische Maßnahmen, die sich an verschiedene Zielgruppen richten, in einen gemeinsamen Handlungsrahmen integriert werden (Vedung 1998, S. 32-33; World Energy Council 2008, S. 11, 106).

Vor dem Hintergrund unserer theoretischen Überlegungen stellen sich folgende Fragen in Bezug auf die Wirkung dieser Instrumente: Lässt sich eine möglichst umfassende Reduktion des Stromverbrauchs privater Haushalte aufgrund der konstatierten akteurs- und situationsbedingten Heterogenität eher durch eine diversifizierte Interventionsstrategie erzielen? Oder unterscheiden sich die Instrumente in ihrer Wirksamkeit sehr deutlich, sodass der fokussierte Einsatz einzelner Instrumente einer kombinierten Anwendung mehrerer Instrumente vorzuziehen ist? Aus diesen Überlegungen ergeben sich folgende Hypothesen:

H1: $\mathrm{Zu}$ deutlichen Stromeinsparungen in privaten Haushalten kommt es nur dann, wenn mehrere politische Instrumente in Kombination eingesetzt werden.

H2: $\mathrm{Zu}$ deutlichen Stromeinsparungen in privaten Haushalten kommt es nur dann, wenn spezifische politische Instrumente für sich genommen eingesetzt werden.

Alternativ zu diesen Annahmen stellt sich die Frage, ob politische Intervention überhaupt eine Rolle bei der Verringerung des Stromkonsums spielt, oder ob diese vielmehr nur durch Kontextfaktoren beeinflusst wird. Eine dritte Hypothese greift dies auf:

H3: Stromeinsparungen in privaten Haushalten sind ausschließlich auf Kontextfaktoren zurückzufübren. 


\section{Methodische Vorgehensweise}

Aufgrund der Überlegung, dass die Maßnahmen und Einflussfaktoren, die zu einem verringerten Stromkonsum privater Haushalte führen, unter Umständen erst bei kombiniertem Einsatz Wirkung entfalten, erscheint die Anwendung einer Qualitative Comparative Analysis (QCA) zur Überprüfung der Hypothesen besonders angebracht. Mithilfe dieser Analysemethode kann untersucht werden, auf welche Bedingungen ein Outcome ${ }^{2}$ zurückgeht (Rihoux u. Grimm 2006, S. 682). QCA räumt dabei auch ganz explizit die Möglichkeit ein, dass Bedingungen kombiniert werden müssen, um ein Outcome zu implizieren (conjunctural causation), bzw. dass das Outcome durch verschiedene Kausalpfade erklärt wird, die einander auch überlappen können (Äquifinalität). Damit stellen Policy-Analysen ein ideales Anwendungsfeld für QCA dar, nachdem auch Policy-Prozesse oftmals auf parallel auftretenden Faktoren beruhen, bzw. nicht nur ein einziges Erklärungsmodell für das Zustandekommen oder den Erfolg einer Policy angegeben werden kann (Scharpf 2006, S. 59). Um diese besonderen Formen komplexer Kausalität korrekt darzustellen, greift QCA auf Konzepte der Mengentheorie, der Booleschen Algebra und der formalen Logik zurück. Die hieraus abgeleiteten Konzepte notwendiger und hinreichender ${ }^{3}$ Bedingungen bilden die Kernelemente einer jeden QCA (Schneider u. Wagemann 2007, S. 31). QCA ist auch dann in der Lage, Kausalbeziehungen aufzuzeigen, wenn keine der Einzelbedingungen eine notwendige oder hinreichende Bedingung für das Outcome darstellt (Schneider u. Wagemann 2007, S. 80). Eine sogenannte INUS-Bedingung liegt beispielsweise dann vor, wenn einzelne Bedingungen, die alleine nicht hinreichend sind (insufficient), in Kombination mit anderen Bedingungen (necessary part of a condition) zu hinreichenden (aber nicht notwendigen) Bedingungen für das Outcome werden ( $u$ nnecessary but sufficient for the result) (Mackie 1974, S. 62; Schneider u. Wagemann 2007, S. 80). ${ }^{4}$

Wir verwenden in dieser Studie die Version der Fuzzy-Set QCA (fsQCA) (Ragin 2008), bei der im Gegensatz zur älteren Crisp-Set-Version die Möglichkeit besteht, die Vollmitgliedschaft eines Falles in einem Konzept (Wert 1) und die absolute Nicht-Mitgliedschaft eines Falles in einem Konzept (Wert 0) quantitativ mit Werten zwischen 0 und 1 zu differenzieren (Ragin 2000, S. 154). Im Bereich der Policy-Forschung, in der häufig mit Konzepten gearbeitet wird, die nicht eindeutig dichotom, aber andererseits auch nicht direkt quantifizierbar sind, erscheint der Einsatz von fsQCA besonders vielversprechend (Wagemann 2008, S. 256). Mithil-

2 Das zu erklärende Phänomen wird im Rahmen von QCA als Outcome und nicht als abhängige Variable bezeichnet, auch um der Unterschiedlichkeit der Kausalmodelle von QCA und statistischen Analysen Rechnung zu tragen. Analog dazu wird eine unabhängige Variable als Bedingung bezeichnet (Schneider u. Wagemann 2007, S. 31-32).

3 In formaler Logik ist die Notation für die Darstellung einer notwendige Bedingung: $\mathrm{X} \leftarrow \mathrm{Y}$. Eine hinreichende Bedingung wird wie folgt dargestellt: $\mathrm{X} \rightarrow \mathrm{Y}$.

4 Beispielsweise stellt $\mathrm{B}$ in der Lösungsformel $\mathrm{A}+\mathrm{BC} \rightarrow \mathrm{Y}$ eine INUS-Bedingung dar, da B für sich genommen keine hinreichende Bedingung ist, sondern ein notwendiger Teil einer kombinierten Bedingung (BC), die dann zwar nicht notwendig ist (A ist die Alternative zu BC), aber in Kombination eine hinreichende Bedingung darstellt. 
fe von Fuzzy-Sets können unscharfe Konzepte formalisiert und präzisere Analysen durchgeführt werden (Verkuilen 2005, S. 464).

\section{Forschungsdesign und Daten}

Unsere Untersuchungsgesamtheit besteht aus 28 Ländern. Diese umfasst zum einen 15 europäische Staaten (Schweden, Dänemark, Tschechien, Großbritannien, Deutschland, Italien, Österreich, Niederlande, Finnland, Frankreich, Polen, Rumänien, Spanien, Estland, Schweiz), die sich hinsichtlich ihrer Größe unterscheiden und verschiedene geografische Zonen Europas abdecken. Zum anderen werden sieben nicht-europäische OECD-Länder (Kanada, Neuseeland, USA, Japan, Australien, Mexiko, Südkorea) berücksichtigt. Mit Brasilien, Indien, China, Argentinien, Singapur und Südafrika gehen zudem auch ausgewählte Schwellenländer in die Untersuchung ein. ${ }^{5}$ Allgemein gesprochen wurden diese 28 Länder ausgewählt, weil sie insgesamt circa 80 Prozent des weltweiten Stromverbrauchs auf sich vereinen. Zudem wird jeder Kontinent durch mindestens einen Staat repräsentiert, so dass verschiedene klimatische Bedingungen berücksichtigt werden können. Der Untersuchungszeitraum umfasst 13 Jahre, von 1995 bis 2007.

Zur Untersuchung unserer Fragestellung wenden wir die von Schneider und Wagemann (2006) entwickelte Zwei-Schritt-Variante von fsQCA an. Dabei wird analytisch zwischen sogenannten nahen und entfernten Einflussfaktoren unterschieden. Entfernte Faktoren zeichnen sich dadurch aus, dass sie nicht oder nur schwer von den handelnden Akteuren beeinflussbar sind. Zugleich sind sie über die Zeit relativ stabil - oftmals handelt es sich sogar um historische Faktoren und haben einen eher indirekten Einfluss auf das Outcome. Daher ist die Analyse der entfernten Faktoren auch zunächst als approximativer Schritt zu verstehen, der dazu dient, die Kontexte festzulegen, in dem die nahen Faktoren wirken.

Die nahen Faktoren sind demgegenüber leichter veränderbar und können von den Akteuren oftmals auch gezielt beeinflusst werden. Zudem stehen sie in einem direkteren Zusammenhang mit dem Outcome, können also eher als die entfernten Faktoren als Kausalmechanismen interpretiert werden (Schneider u. Wagemann 2006, S. 760). Damit wird der Zwei-Schritt-Ansatz den tatsächlichen Kausalbeziehungen gerechter, als dies bei undifferenzierter gleichzeitiger Verwendung aller Faktoren geschieht. Es ist zudem ein angenehmer Nebeneffekt dieses Vorgehens, dass eine verhältnismäßig größere Anzahl an Bedingungen in die Analyse einbezogen werden kann, nachdem diese in zwei Teile aufgeteilt wird. Dies wirkt sich wiederum positiv auf das Problem der begrenzten empirischen Vielfalt ${ }^{6}$ (Ragin 1987, S. 104-113; Schneider u. Wagemann 2007, S. 101-109) aus. Eine solche Zwei-Schritt-Analyse erscheint auch in unserem Fall besonders geeignet, nachdem

5 Daten wurden auch zu Russland und Chile erhoben, jedoch wurden diese beiden Länder nicht in die Analyse aufgenommen, da im Falle Russlands starke Inkonsistenzen hinsichtlich der Entwicklung der Stromverbrauchswerte zu beobachten waren. In Chile hingegen wurden politische Maßnahmen erst im Jahr 2007 eingeführt, sodass deren Effekt noch nicht erfasst werden kann.

6 Damit wird in QCA die Situation beschrieben, dass es für formal-logisch mögliche Kombinationen von Bedingungen keine empirischen Fälle gibt. Auch bei QCA muss die Fallzahl in einem vernünftigen Verhältnis zur Anzahl der Erklärungsfaktoren stehen. 
einerseits politische Maßnahmen, aber andererseits eben auch Kontextfaktoren das Auftreten des Outcomes begünstigen können. Somit stellen die eingesetzten Politiken in unserer Analyse die nahen Faktoren dar, während die wirtschaftliche Entwicklung, das Klima und Urbanisierung (als Proxy für sozioökonomische Modernisierung) als entfernte Faktoren berücksichtigt werden.

\subsection{Outcome: Rückgang des Stromverbrauchs}

Um zu bestimmen, ob es zu einer Verringerung des Stromverbrauchs privater Haushalte kam, werden die Wachstumsraten des Stromverbrauchs pro Kopf, ausgehend vom Jahr 1995, verwendet. Für die Daten des Pro-Kopf-Stromverbrauchs privater Haushalte wird auf die Datenbanken der Vereinten Nationen zurückgegriffen.

Dabei werden hohe Fuzzy-Werte an all jene Länder vergeben, in denen es zu einem Rückgang des Stromkonsums kam - erfasst wird also der Erfolg der Maßnahmen. Mithilfe der direkten Methode der Kalibrierung ${ }^{7}$ werden den Werten, die aus der Berechnung der Wachstumsrate hervorgehen, Fuzzy-Werte zugeteilt. Dafür müssen zuerst Schwellenwerte für die volle Mitgliedschaft, den CrossoverPoint und die volle Nichtmitgliedschaft in der Menge der Länder, die ihren Stromkonsum reduziert haben, bestimmt werden. Der Schwellenwert für die volle Mitgliedschaft wird als Wachstumsrate 0 definiert: Alle Länder, die kein Wachstum (oder gar ein Minuswachstum) des Stromverbrauchs pro Kopf aufweisen, sollen also als volle Mitglieder in der Menge der stromsparenden Länder gelten. Ferner sollen alle Länder, die eine durchschnittliche jährliche Wachstumsrate von über zwei Prozent aufweisen, über dem Crossover-Point liegen, alle anderen darunter. Der Grund hierfür ist, dass Wachstumsraten des Stromverbrauchs privater Haushalte von ein bis zwei Prozent pro Jahr nach herrschender Meinung einen immer noch relativ moderaten Anstieg des Stromkonsums darstellen (World Energy Council 2008, S. 31), wie er selbst bei konsequenter Verfolgung nationaler Einsparziele aufgrund gegenläufiger Tendenzen wie dem Rebound-Effekt ${ }^{8}$ oftmals kaum vermieden werden kann. Der Crossover-Point wird demnach bei einer Gesamtwachstumsrate von 26 Prozent angesetzt (13 jährliche Steigerungen um zwei Prozent). Hinsichtlich der vollen Nichtmitgliedschaft im Konzept soll nur China einen Fuzzy-Wert von 0 erhalten, da sich China extrem deutlich durch eine überdurchschnittlich hohe Wachstumsrate (230,12 Prozent) von den anderen Ländern absetzt. Das Land mit der zweithöchsten Wachstumsrate (95,58 Prozent) soll da-

7 Bei der direkten Methode der Kalibrierung werden die Werte einer quantitativen (intervallskalierten) Variable durch einen halb-automatisierten Prozess in Fuzzy-Werte kalibriert, indem Schwellenwerte für die Fuzzy-Werte 0,95 (als Proxy für Vollmitgliedschaft), 0,05 (Nicht-Mitgliedschaft) und 0,50 (für den Crossover-Point) theoriegeleitet festgelegt werden. Auf Basis dieser Werte werden anschließend mit Logit-Funktionen für die verbleibenden empirisch beobachtbaren Fälle Fuzzy-Werte errechnet. Diese Methode gilt als Alternative zu hauptsächlich theoriegeleiteten Kalibrierungsmethoden und kann bei eindeutig quantitativen Ausgangsdaten angewandt werden (für Einzelheiten zur direkten Methode siehe Ragin 2008, S. 85-108).

8 Als Rebound-Effekt wird der Zuwachs des privaten Energieverbrauchs trotz Effizienzsteigerungen bezeichnet, der vor allem der kontinuierlichen Ausweitung des Gerätebestands geschuldet ist (Sorrell 2007). 
gegen gerade unter dem Schwellenwert für volle Nicht-Mitgliedschaft liegen. Dieser wird also mit 96 Prozent festgelegt.

Tabelle 1: Kalibrierungstabelle - Outcome

\begin{tabular}{|c|c|c|c|}
\hline \multirow[t]{2}{*}{ Land (Akronym) } & \multicolumn{2}{|c|}{ Wachstumsrate } & \multirow[t]{2}{*}{ Fuzzy-Wert } \\
\hline & $1995-2007$ & pro Jahr & \\
\hline Schweden (SE) & $-8,08$ & $-0,70$ & 0,98 \\
\hline Dänemark (DK) & $-5,49$ & $-0,47$ & 0,97 \\
\hline Tschechien (CZ) & $-0,42$ & $-0,03$ & 0,95 \\
\hline \multicolumn{4}{|c|}{0 als Schwellenwert für volle Mitgliedschaft $(=0,95)$} \\
\hline Neuseeland (NZ) & 6,05 & 0,49 & 0,91 \\
\hline Kanada (CA) & 6,07 & 0,49 & 0,91 \\
\hline Großbritannien (UK) & 8,23 & 0,66 & 0,89 \\
\hline Schweiz $(\mathrm{CH})$ & 8,69 & 0,70 & 0,88 \\
\hline Deutschland (DE) & 9,11 & 0,73 & 0,88 \\
\hline Österreich (AT) & 14,80 & 1,16 & 0,78 \\
\hline Niederlande (NL) & 15,07 & 1,18 & 0,78 \\
\hline Italien (IT) & 15,72 & 1,22 & 0,77 \\
\hline USA (USA) & 18,15 & 1,40 & 0,71 \\
\hline Brasilien (BR) & 20,57 & 1,57 & 0,65 \\
\hline Japan (JP) & 20,97 & 1,60 & 0,64 \\
\hline Frankreich $(\mathrm{FR})$ & 25,56 & 1,92 & 0,51 \\
\hline \multicolumn{4}{|c|}{26 als Crossover-Point $(=0,5)$} \\
\hline Finnland (FI) & 28,82 & 2,13 & 0,47 \\
\hline Australien (AU) & 31,54 & 2,31 & 0,44 \\
\hline Argentinien (AR) & 47,31 & 3,28 & 0,29 \\
\hline Polen $(\mathrm{PL})$ & 47,65 & 3,30 & 0,28 \\
\hline Südafrika $(\mathrm{ZA})$ & 47,66 & 3,30 & 0,28 \\
\hline Rumänien (RO) & 48,41 & 3,34 & 0,28 \\
\hline Mexiko (MX) & 50,00 & 3,44 & 0,26 \\
\hline Singapur (SG) & 57,52 & 3,86 & 0,21 \\
\hline Südkorea (KR) & 79,11 & 4,98 & 0,09 \\
\hline Estland (EE) & 82,52 & 5,14 & 0,08 \\
\hline Indien (IN) & 91,07 & 5,54 & 0,06 \\
\hline Spanien (ES) & 95,58 & 5,75 & 0,05 \\
\hline \multicolumn{4}{|c|}{96 als Schwellenwert für volle Nichtmitgliedschaft $(=0,05)$} \\
\hline China $(\mathrm{CN})$ & 230,12 & 10,46 & 0 \\
\hline
\end{tabular}

\subsection{Nahe Faktoren}

Im Rahmen des Forschungsverbundprojekts Transpose wurden Daten zu PolicyMaßnahmen in den Ländern erhoben, die in unsere Analyse eingehen. Hier werden alle diejenigen Maßnahmen berücksichtigt, die jeweils auf nationaler Ebene im Zeitraum von 1995 bis 2007 implementiert wurden bzw. in diesen Zeitraum hineingewirkt haben. Diese Maßnahmen wurden den vier Instrumentenkategorien zugeteilt. Zur Kategorie der ökonomischen Anreize wurden progressive Strompreise, Verbrauchssteuern, Steuerermäßigungen, Kredite mit niedrigem Zinssatz 
und Subventionen sowie Bonusprogramme gezählt. Beispielhaft sind in diesem Zusammenhang zinsgünstige Kredite und Zuschüsse zu nennen, die für energetische Sanierungen von Wohngebäuden gewährt werden.

Die Kategorie informativer Instrumente umfasst Informations- und Werbekampagnen, Beratungsangebote und Bildungsmaßnahmen, z. B. an Schulen, sowie Informationsvermittlung über Internetseiten und Informationsbroschüren. Auch informative Stromrechnungen werden berücksichtigt. Diese geben detaillierte Auskunft darüber, wann wie viel Strom verbraucht wurde, oder liefern Vergleichswerte zum Vorjahr bzw. zum Stromkonsum ähnlicher Haushalte (Duscha 2008, S. 154). Im Gegensatz zu konventionellen Stromrechnungen, in denen lediglich der jährliche Stromverbrauch dargestellt wird, ermöglichen es informative Stromrechnungen den Verbrauchern, ihren Stromverbrauch besser nachverfolgen und einordnen zu können.

All jene Maßnahmen, die festlegen, dass Elektrogeräte mit EnergieeffizienzLabeln ausgestattet bzw. bestimmte Energieeffizienz-Standards eingehalten werden müssen, werden der Instrumentenkategorie Normen und Standards zugeteilt. Standards legen fest, welchen Energieeffizienz-Kriterien elektrische Geräte genügen müssen. Eine Sonderrolle fällt in dieser Hinsicht dem in Japan verfolgten dynamischen Top-Runner-Ansatz zu, bei dem sich der jeweils geltende Standard am energieeffizientesten Produkt orientiert, das auf dem Markt erhältlich ist (siehe dazu Nordqvist 2006).

Die Unterschiede im Stromverbrauch einer Geräteart können zudem bildhaft mit Energieeffizienz-Labeln kenntlich gemacht werden. Innerhalb der Europäischen Union werden beispielsweise Effizienzklassen von A bis G verwendet (mittlerweile ergänzt durch $\mathrm{A}+$ bis $\mathrm{A}+++$ ), die farblich abgestuft sind. Der Konsument kann sich somit schnell ein Urteil über den Energieverbrauch eines Gerätes bilden, und der Vergleich zwischen konkurrierenden Geräten wird erheblich erleichtert.

Auch Programme, die festlegen, dass bestimmte Produkte mit einem hohen Energieverbrauch vom Markt genommen werden müssen (sogenanntes Phaseout), fallen in die Kategorie der Normen und Standards. In einigen Ländern gelten zudem Energie-Standards, die beim Bau oder der Sanierung von Wohngebäuden eingehalten werden müssen. Vereinzelt besteht auch die Pflicht, die Energieeffizienz von Wohngebäuden zu erfassen und zu dokumentieren.

Unter die Kategorie der Institutionen wurden in erster Linie Energieagenturen und Energieeffizienzfonds subsumiert. Zusätzlich wurden staatliche Komitees, halbstaatliche Organisationen und Nichtregierungsorganisationen berücksichtigt, die sich der Förderung von Energieeffizienz widmen, insofern die Aufgaben dieser Institutionen mit denen von Energieeffizienzfonds und Energieagenturen vergleichbar sind. Allen genannten Einrichtungen ist gemein, dass sie übergreifende Handlungskonzepte entwickeln und diese unter Einsatz verschiedener, aufeinander abgestimmter Maßnahmen umsetzen bzw. die Umsetzung unterstützend begleiten. Die folgende Tabelle gibt einen Überblick über die verschiedenen Instrumententypen. Deren jeweilige idealtypische Wirkungsweise wird dabei nochmals zusammenfassend dargestellt. Beispiele konkreter Maßnahmen sollen zu einer besseren Verständlichkeit beitragen. 


\section{Tabelle 2: Instrumententypen und idealtypische Wirkungsweise}

\begin{tabular}{|c|c|c|}
\hline Instrumententyp & Wirkungsweise & Beispiele (Land) \\
\hline $\begin{array}{l}\text { Ökonomische An- } \\
\text { reize }\end{array}$ & $\begin{array}{l}\text { Positive oder negative finanzi- } \\
\text { elle Anreize sollen Verhaltens- } \\
\text { änderungen der Verbraucher } \\
\text { bewirken. }\end{array}$ & $\begin{array}{l}\text { Abgaben oder Steuern auf Stromkonsum } \\
\text { (Österreich, Dänemark, Finnland, } \\
\text { Deutschland, Niederlande, Großbritanni- } \\
\text { en); flexible Stromtarife (China, Frank- } \\
\text { reich); Zuschüsse und Steuervergünsti- } \\
\text { gungen für den Erwerb energieeffizienter } \\
\text { Produkte wie Wärmepumpen, etc., für } \\
\text { Maßnahmen im Rahmen energetischer } \\
\text { Sanierungen oder für die Durchführung } \\
\text { einer Energiediagnose (weit verbreitet) }\end{array}$ \\
\hline Infor & $\begin{array}{l}\text { Sensibilisierung und Bewusst- } \\
\text { seinsschaffung für Einspar- } \\
\text { möglichkeiten soll Verhaltens- } \\
\text { änderungen bewirken. }\end{array}$ & $\begin{array}{l}\text { Informative Stromrechnung (Japan, Aus- } \\
\text { tralien); Web-Tool zur Analyse und Kon- } \\
\text { trolle des individuellen Stromverbrauchs } \\
\text { (Dänemark, Österreich); Internetseite mit } \\
\text { Auskunft über besonders energieeffizien- } \\
\text { te Produkte (Schweiz, Deutschland) }\end{array}$ \\
\hline $\begin{array}{l}\text { Normen und Stan- } \\
\text { dards }\end{array}$ & $\begin{array}{l}\text { Marktdurchdringung energie- } \\
\text { effizienter Geräte soll be- } \\
\text { schleunigt und Transaktions- } \\
\text { kosten für Informations- } \\
\text { beschaffung sollen gesenkt } \\
\text { werden. }\end{array}$ & $\begin{array}{l}\text { EU-Label zur Energieverbrauchskenn- } \\
\text { zeichnung (EU-Mitgliedsländer); EU- } \\
\text { Ökodesign-Richtlinie zur Verbesserung } \\
\text { der Energieeffizienz von Elektrogeräten } \\
\text { (EU-Mitgliedsländer, weit verbreitet in } \\
\text { übrigen Untersuchungsländern, in der } \\
\text { Schweiz, in Singapur und Südafrika je- } \\
\text { doch nur marginal) }\end{array}$ \\
\hline Institutionen & $\begin{array}{l}\text { Ein übergreifender Hand- } \\
\text { lungsrahmen soll die koordi- } \\
\text { nierte Anwendung verschiede- } \\
\text { ner Maßnahmen ermöglichen. }\end{array}$ & $\begin{array}{l}\text { Energieagenturen, Energieeffizienzfonds } \\
\text { und vergleichbare Institutionen, die sich } \\
\text { der Förderung von Energieeffizienz wid- } \\
\text { men (weit verbreitet) }\end{array}$ \\
\hline
\end{tabular}

Eine mögliche Überlegung hinsichtlich der geeigneten Vorgehensweise, die Wirkung der unterschiedlichen Instrumente abzubilden, wäre die Aufsummierung der verschiedenen Einzelmaßnahmen, die von den jeweiligen Ländern innerhalb des Untersuchungszeitraums implementiert wurden. Diese Überlegung würde auf der Annahme basieren, dass sich das Aggregat der unterschiedlichen Maßnahmen als Näherungswert für deren Intensität eignet. Diese Vorgehensweise erscheint jedoch problematisch, da sie die spezifische Wirkung einzelner Maßnahmen unberücksichtigt lässt, wie beispielsweise physikalische Effekte. Die Tatsache, dass die Einführung von Effizienzstandards für Kühlschränke rein physikalisch eine größere Wirkung zu erzeugen vermag als Effizienzstandards für Lampen, kann auf diese Weise nicht berücksichtigt werden. Das gleiche gilt für die zeitliche Dimension, d. h. ob beispielsweise ein Land seit mehreren Jahren über eine Energieeffizienzagentur verfügt oder erst seit kurzem.

Demgegenüber ermöglichen Indizes, in denen einzelne Maßnahmen je nach Anzahl, Dauer und Intensität unterschiedlich gewichtet werden, eine adäquatere Berücksichtigung jeder Maßnahme. Zur Gewichtung der Intensität der jeweiligen politischen Maßnahmen wurde auf Statistiken zurückgegriffen, die den durchschnittlichen Stromverbrauch einzelner Geräte angeben. Für jeden Maßnahmetyp 
wurden die verschiedenen Dimensionen, d. h. Anzahl, Intensität und Zeitrahmen in Ordinalskalen abgebildet. Diese Dimensionen wurden anschließend mithilfe des Partial-Order-Scaling-Verfahrens, das im Programmpaket SYSTAT im Verfahren POSAC implementiert ist, in einzelne Politikinstrument-Indizes integriert (jeweils für Institutionen, Normen und Standards, finanzielle Anreize und Informationen) (zur Methode siehe Tenbücken u. Thiem 2004).

\subsection{Entfernte Faktoren}

Wirtschaftswachstum: Ein sich positiv entwickelndes Wirtschaftswachstum gilt als einer der Haupteinflussfaktoren für ansteigenden Konsum (OECD 2008a, S. 48). Bezogen auf den Stromverbrauch privater Haushalte ist anzunehmen, dass mit zunehmendem Wohlstandsniveau die Nachfrage nach energieintensiven Konsumgütern zunimmt. Der Zusammenhang zwischen der Wirtschaftsleistung eines Landes und dessen Energiekonsum ist bereits in vielen Studien nachgewiesen worden (Kraft u. Kraft 1978; Schipper u. Grubb 2000; Soytas u. Sari 2003). Zur Messung der Wirtschaftsleistung verwenden wir das reale Bruttoinlandsprodukt pro Kopf aus dem Penn World Table. Um zu erfassen, wie sich die Wirtschaftsleistung über den gesamten Untersuchungszeitraum entwickelt hat, wurde die Wachstumsrate des BIP im Zeitraum von 1995 bis 2007 berechnet. Die Kalibrierung wurde so vorgenommen, dass jene Länder, die geringe Wachstumsraten des Bruttoinlandsprodukts aufweisen, relativ hohe Fuzzy-Werte erhalten. In diesem Fall greifen wir auf die indirekte Methode der Kalibrierung ${ }^{9}$ zurück, zumal die für die direkte Methode notwendigen Schwellenwerte schwer zu definieren sind. ${ }^{10}$

Klima: Auch das durchschnittliche Landesklima gilt als Einflussfaktor für den unterschiedlichen Stromverbrauch privater Haushalte (IEA 2004, S. 88). In Studien, die klimatische Unterschiede zwischen Ländern oder im Jahresverlauf innerhalb eines Landes berücksichtigen, konnte ein Zusammenhang zwischen klimatischen Verhältnissen und der Nachfrage nach Energie bestätigt werden (Henley u. Peirson 1998; Metcalf 2008; Reiss u. White 2008). Je mehr die klimatischen Bedingungen in einem Land im Jahresverlauf von einer dem Wohnkomfort angemessenen Temperatur abweichen, desto eher ist mit einem erhöhten Stromverbrauch zu rechnen, der für Kühl- oder Heizfunktionen aufgebracht wird. In

9 Für die indirekte Methode der Kalibrierung müssen quantitative (intervallskalierte) Daten vorliegen. Im Unterschied zur direkten Methode, bei der drei qualitative Schwellenwerte definiert werden, werden hier die Fälle mithilfe theoretischer Kriterien grob in die folgenden sechs Fuzzy-Werte-Kategorien eingeteilt: 1 („das Konzept liegt voll vor“), 0,8 („,das Konzept liegt vor“), 0,6 („das Konzept liegt eher vor als nicht“), 0,4 („,das Konzept liegt eher nicht vor als dass es vorliegt“), 0,2 („das Konzept liegt vor“) und 0 („,das Konzept liegt überhaupt nicht vor“). Danach werden diese Werte durch ein fraktioniertes Logit-Modell neu skaliert (Funktion FRACPOLY in STATA), sodass wieder intervallskalierte Werte vorliegen, die aber zwischen 0 und 1 liegen (für weitere technische Details, siehe Ragin 2008, S. 84, 94). Da sie auf einer ersten theoriegestützten Vor-Gruppierung beruhen, kann davon ausgegangen werden, dass diese neu skalierten Werte die dahinter liegenden Konzepte gut repräsentieren.

10 Korrelationsanalysen legen den Schluss nahe, dass das BIP als Indikator für Wirtschaftsentwicklung auch bestehende sozio-demografische und technische Unterschiede widerspiegelt und daher stellvertretend als Kontrollgröße verwendet werden kann. Auf diese Weise kann die Zahl der zu berücksichtigenden Kontextfaktoren gering gehalten werden. 
gemäßigten Klimazonen sollte demnach der Stromverbrauch geringer sein als in klimatisch extremeren Zonen.

Da für unsere Untersuchungseinheit mit Ausnahme der EU-Staaten und den USA keine vergleichbaren Daten vorliegen, wurden mittels aufwändiger Datenrecherchen eigene Wetterzeitreihen zusammengestellt. Mithilfe der Daten des National Climatic Data Center und der National Oceanic and Atmospheric Administration wurden für alle Untersuchungsländer die durchschnittlichen Tagestemperaturen für die Jahre 1995 bis 2007 errechnet. In den meisten Fällen wurden dafür die Temperaturwerte einer großen, im Zentrum des Landes gelegenen Stadt verwendet. Für größere Länder wie die USA, Kanada, Australien, China, Indien und Südafrika wurden aufgrund der unterschiedlichen Klimazonen zwei oder mehrere Städte ausgewählt und entsprechend der Größe und Population der entsprechenden Region gewichtet (Schätzwerte). Fehlende Werte wurden interpoliert bzw. mit Werten des Vorjahres ersetzt. Die auf diese Weise gewonnenen Daten dienen als Basis für die Berechnung von Heiz- und Kühlgradtagen. Diese Einheiten geben an, an wie vielen Tagen im Jahr die tatsächliche Temperatur von einer Basistemperatur, die auf 18 bzw. 21 Grad Celsius festgelegt ist, nach unten bzw. nach oben abweicht. ${ }^{11}$ Abschließend wurde das arithmetische Mittel der Summe aus Heiz- und Kühlgradtagen errechnet, die ein Land im Zeitraum zwischen 1995 und 2007 aufweist.

Selbstverständlich dienen die errechneten Werte lediglich als Proxies für die klimatischen Verhältnisse in den Untersuchungsländern, nicht allein aufgrund der Tatsache, dass die unterschiedlichen Subklimata in den einzelnen Ländern nur annäherungsweise Berücksichtigung finden. Darüber hinaus ist anzunehmen, dass der Stromverbrauch nicht linear mit der Außentemperatur steigt und fällt. Zudem spiegeln Temperaturdaten nicht das tatsächliche Temperaturempfinden wider; weitere Einflussgrößen sind Sonneneinstrahlung und Luftfeuchtigkeit. Nicht zuletzt spielen auch soziokulturelle Unterschiede beim Temperaturempfinden eine Rolle. Trotz dieser Einschränkung liefern die errechneten Heiz- und Kühlgradtage eine gute Annäherung an das Konzept der klimatischen Verhältnisse.

Die Kalibrierung wurde so vorgenommen, dass jene Länder, in denen gemäßigte klimatische Verhältnisse herrschen, hohe Fuzzy-Werte erhalten. Dabei wurde wiederum die indirekte Methode der Kalibrierung angewandt.

Urbanisierung: Schließlich können auch Urbanisierungstendenzen zur Erklärung des Stromkonsums im Haushaltsbereich beitragen (OECD 2008b). Insbesondere in Schwellenländern sollte der Zuwachs an in Städten lebenden Menschen mit einem höheren Strombedarf einhergehen. Tendenziell höhere Fuzzy-Werte erhalten all jene Länder, in denen der Zuwachs an in Städten lebenden Menschen gering ausgefallen ist. Um die Entwicklung der Urbanisierung zu messen, stützen wir uns auf die World Development Indicators der Weltbank und berechnen die prozentuale Veränderung der städtischen Bevölkerung innerhalb des Untersuchungszeitraums, die dann mit der indirekten Methode der Kalibrierung in Fuzzy-Werte transformiert werden.

11 Ein Vergleich der aus unseren Daten gewonnenen Heizgradtage der EU-Länder mit den EurostatDaten ergibt einen Korrelationswert (Pearson) von 0,91. 
Diese drei Bedingungen können als ferne Faktoren im Sinne des oben genannten Zwei-Schritt-Ansatzes gelten. Sie sind über die Zeit relativ stabil und beispielsweise durch politische Faktoren wenig bis gar nicht beeinflussbar. Sie haben aber einen Effekt auf das Outcome. Wir gehen also davon aus, dass diese fernen Faktoren den strukturellen Kontext bilden, der für den Stromverbrauch privater Haushalte von Bedeutung ist und dass diese Faktoren daher berücksichtigt werden müssen.

In der folgenden Übersicht sind alle wichtigen Kriterien der Konzeptspezifikation und Kalibrierung zusammenfassend dargestellt.

Tabelle 3: Übersicht über Outcome, Bedingungen und Kontextbedingungen

\begin{tabular}{|c|c|c|c|c|c|c|}
\hline & $\begin{array}{l}\text { Bezeich- } \\
\text { nung }\end{array}$ & $\begin{array}{l}\text { Umgang } \\
\text { mit zeitli- } \\
\text { cher Kom- } \\
\text { ponente }\end{array}$ & $\begin{array}{l}\text { Methode } \\
\text { der Kali- } \\
\text { brierung }\end{array}$ & $\begin{array}{l}\text { Kriterien der } \\
\text { Kalibrierung }\end{array}$ & $\begin{array}{l}\text { Definition } \\
\text { hoher Fuzzy- } \\
\text { Werte }\end{array}$ & $\begin{array}{l}\text { Primäre } \\
\text { Datenquelle }\end{array}$ \\
\hline \multicolumn{7}{|l|}{ Outcome } \\
\hline $\begin{array}{l}\text { Rückgang } \\
\text { des Strom- } \\
\text { konsums }\end{array}$ & $\begin{array}{l}\text { elco (elec- } \\
\text { tricity } \\
\text { consump- } \\
\text { tion) }\end{array}$ & $\begin{array}{l}\text { Wachs- } \\
\text { tumsrate } \\
\text { von } 1995 \\
\text { bis } 2007 \\
\end{array}$ & $\begin{array}{l}\text { Direkte } \\
\text { Methode }\end{array}$ & $\begin{array}{l}\text { Schwellen- } \\
\text { werte: } \\
0,26,96\end{array}$ & $\begin{array}{l}\text { Rückgang } \\
\text { des Strom- } \\
\text { verbrauchs }\end{array}$ & UN \\
\hline \multicolumn{7}{|c|}{ Nahe Faktoren } \\
\hline $\begin{array}{l}\text { Ökonomi- } \\
\text { sche An- } \\
\text { reize }\end{array}$ & eco & \multirow{4}{*}{$\begin{array}{l}\text { Berück- } \\
\text { sichtigung } \\
\text { aller Inst- } \\
\text { rumente, } \\
\text { die im } \\
\text { Zeitraum } \\
\text { von } 1995 \\
\text { bis } 2007 \\
\text { wirksam } \\
\text { waren }\end{array}$} & \multirow[t]{4}{*}{$\begin{array}{l}\text { Theorie- } \\
\text { geleitet }\end{array}$} & \multirow{4}{*}{$\begin{array}{l}\text { Anzahl, } \\
\text { Dauer und } \\
\text { Intensität } \\
\text { der Maßnah- } \\
\text { men }\end{array}$} & \multirow[t]{4}{*}{$\begin{array}{l}\text { Hohe politi- } \\
\text { sche Aktivi- } \\
\text { tät }\end{array}$} & \multirow{4}{*}{$\begin{array}{l}\text { Transpose-Da- } \\
\text { tenbank, Da- } \\
\text { ten erhoben im } \\
\text { Rahmen des } \\
\text { Transpose- } \\
\text { Forschungs- } \\
\text { projekts }\end{array}$} \\
\hline $\begin{array}{l}\text { Informa- } \\
\text { tionen }\end{array}$ & info & & & & & \\
\hline $\begin{array}{l}\text { Normen } \\
\text { und Stan- } \\
\text { dards }\end{array}$ & norm & & & & & \\
\hline $\begin{array}{l}\text { Institu- } \\
\text { tionen }\end{array}$ & ag & & & & & \\
\hline \multicolumn{7}{|c|}{ Entfernte Faktoren } \\
\hline $\begin{array}{l}\text { Wirtschaft- } \\
\text { liche Leis- } \\
\text { tung }\end{array}$ & bip & $\begin{array}{l}\text { Wachs- } \\
\text { tumsrate } \\
\text { von } 1995 \\
\text { bis } 2007 \\
\end{array}$ & \multirow[t]{3}{*}{$\begin{array}{l}\text { Indirekte } \\
\text { Methode }\end{array}$} & \multirow{3}{*}{$\begin{array}{l}\text { Relation der } \\
\text { Fälle zuein- } \\
\text { ander, dem- } \\
\text { entsprechend } \\
\text { Einteilung in } \\
\text { sechs Kate- } \\
\text { gorien }\end{array}$} & $\begin{array}{l}\text { Geringe } \\
\text { Wachstums- } \\
\text { raten des BIP }\end{array}$ & $\begin{array}{l}\text { Penn World } \\
\text { Table }\end{array}$ \\
\hline Klima & klima & $\begin{array}{l}\text { Arithmeti- } \\
\text { sches Mit- } \\
\text { tel der } \\
\text { Werte zwi- } \\
\text { schen } 1995 \\
\text { und } 2007\end{array}$ & & & $\begin{array}{l}\text { Gemäßigtes } \\
\text { Klima }\end{array}$ & $\begin{array}{l}\text { National Oce- } \\
\text { anic and At- } \\
\text { mospheric Ad- } \\
\text { ministration } \\
\text { und National } \\
\text { Climatic Data } \\
\text { Center }\end{array}$ \\
\hline $\begin{array}{l}\text { Urbanisie- } \\
\text { rung }\end{array}$ & urb & $\begin{array}{l}\text { Wachs- } \\
\text { tumsrate } \\
\text { von } 1995 \\
\text { bis } 2007\end{array}$ & & & $\begin{array}{l}\text { Geringes } \\
\text { Ausmaß an } \\
\text { Urbanisie- } \\
\text { rung }\end{array}$ & $\begin{array}{l}\text { World Deve- } \\
\text { lopment Indi- } \\
\text { cators der } \\
\text { Weltbank }\end{array}$ \\
\hline
\end{tabular}




\section{Analyse}

\subsection{Erster Analyseschritt}

Ziel des ersten Analyseschrittes ist es, zwischen mehreren potenziellen Kontextfaktoren diejenigen auszumachen, innerhalb derer das Auftreten des Outcomes leichter möglich ist. Es handelt sich demnach um einen ergebnisumschreibenden Schritt, welcher der Vorbereitung des ergebnispräzisierenden zweiten Schritts dient (Schneider/Wagemann 2007, S. 260).

In den ersten Analyseschritt werden die Kontextbedingungen der Wachstumsraten der Wirtschaftsleistung, der klimatischen Verhältnisse und der Entwicklung der Urbanisierung aufgenommen. Im Fall von drei Bedingungen ergeben sich $2^{3}$ logisch mögliche Kombinationen, die in Form einer achtzeiligen Wahrheitstafel dargestellt werden können (Ragin 2008, S. 124-125). ${ }^{12}$ Jeder Fall kann, je nach Ausprägung der Fuzzy-Werte in den Bedingungen, einem Idealtypen, das heißt einer spezifischen Konfiguration von Bedingungen zugeordnet werden, wie in Tabelle 4 verdeutlicht wird (vgl. dazu Schneider u. Wagemann 2007, S. 195). Obwohl die Darstellung der Idealtypen eine Dichotomisierung suggeriert, bleiben die spezifischen Informationen über die Fuzzy-Werte der einzelnen Fälle jedoch erhalten und werden für die folgenden analytischen Schritte herangezogen, um den Lösungsterm und seine Güte zu bestimmen (Schneider u. Wagemann 2007, S. 86-87). Die Konsistenz- und Abdeckungsmaße geben an, wie gut eine Erklärung ist bzw. wie viel erklärt werden kann (Blatter et al. 2007, S. 225).

\section{Tabelle 4: Wahrheitstafel - Erster Analyseschritt}

\begin{tabular}{|lllllll|}
\hline klima & bip & urb & N & elco & consistency & Land \\
\hline 1 & 1 & 1 & 5 & 1 & 0,87 & DK, DE, AT, SE, CH \\
0 & 1 & 1 & 4 & 1 & 0,87 & IT, JP, NZ, UK \\
1 & 1 & 0 & 1 & 1 & 0,86 & CA \\
0 & 1 & 0 & 8 & 1 & 0,77 & AR, AU, BR, FR, ZA, USA, MX, NL \\
1 & 0 & 0 & 1 & 0 & 0,71 & KR \\
0 & 0 & 1 & 2 & 0 & 0,71 & SG, ES \\
0 & 0 & 0 & 2 & 0 & 0,67 & CN, IN \\
1 & 0 & 1 & 5 & 0 & 0,66 & EE, FI, PL, RO, CZ \\
\hline \multicolumn{7}{|l}{ N = Anzahl der Fälle, die dem Idealtyp zugeordnet werden können } \\
\hline
\end{tabular}

Mithilfe des Truth-Table-Algorithmus (Ragin 2008, S. 124-146) erfolgt die Minimierung der Wahrheitstafel. Dabei wurde als Cut-Off-Point (Schneider u. Wage-

12 Die Kombinationen ergeben sich aus der Tatsache, dass die einzelnen Bedingungen jeweils vorliegen können oder auch nicht. 
mann 2007, S. 222) ein Konsistenzmaß von 0,75 festgelegt ${ }^{13}$; eine Entscheidung hinsichtlich der Behandlung empirisch begrenzter Vielfalt ist nicht zu treffen, da alle logisch möglichen Kombinationen auch empirisch vorliegen. Folgender Lösungsterm ergibt sich:

Tabelle 5: Ergebnis erster Analyseschritt

$$
\begin{aligned}
& \text { bip } \rightarrow \text { elco } \\
& \text { Abdeckung: 0,84; Konsistenz: 0,76 }
\end{aligned}
$$

Von allen drei Kontextbedingungen findet sich im Lösungsterm des ersten Analyseschritts allein die Wirtschaftsleistung. Mit einem Abdeckungsmaß von 0,84 und einem Gesamtkonsistenzwert von 0,76 weist dieser Lösungsterm bereits eine für den ersten Analyseschritt recht hohe Güte auf - Schneider und Wagemann schlagen in der Tat vor, beim ersten Schritt niedrigere Konsistenzwerte als gewöhnlich zu akzeptieren (Schneider u. Wagemann 2006, S. 77). Bei der Wirtschaftsleistung handelt es sich also um einen sehr einflussreichen Kontextfaktor. Im zweiten Analyseschritt wird nun untersucht, ob die nahen Faktoren im Kontext der Wirtschaftsleistung Wirkung zeigen.

\subsection{Zweiter Analyseschritt}

Zur Überprüfung unserer Hypothese, dass nur durch eine Kombination verschiedener politischer Instrumente Stromeinsparungen in privaten Haushalten zu erzielen sind, wird zunächst jede einzelne Bedingung daraufhin geprüft, ob sie notwendig ist für das Auftreten des Outcomes. Lägen notwendige Bedingungen vor, so würde das bedeuten, dass ein einzelnes politisches Instrument unabdingbar für das Outcome ist. Tabelle 6 gibt die Ergebnisse wieder.

13 Die Konsistenzmaße in einer Wahrheitstafel geben an, inwieweit die Daten die Aussage unterstützen, dass eine gegebene Kombination von Bedingungen eine hinreichende Bedingung für das Outcome darstellt. In fsQCA sind voll konsistent hinreichende Bedingungen (mit einem Konsistenzwert von 1) aber sehr selten. Um den Lösungsterm zu berechnen, der das Resultat der logischen Minimierung aller als hinreichend identifizierten Bedingungen ist, muss ein sogenannter Cut-OffPoint bestimmt werden, der festlegt, bis zu welchem Konsistenzwert eine hinreichende Bedingung noch als akzeptabel gilt und in die Minimierung einbezogen werden kann (Schneider u. Wagemann 2007, S. 222). Dabei gibt es keine allgemeinverbindlichen Regeln, welcher Wert dies sein soll, auch wenn für hinreichende Bedingungen der Wert von 0,75 verschiedentlich erwähnt wird (Ragin 2008, S. 118; Schneider u. Wagemann 2010, S. 407).

Im Falle des Zwei-Schritt-Ansatzes - der ja bewusst flexibel sein soll, und bei dem die Kontexte als ergebnisermöglichende Faktoren eher breit angelegt sind - wird im ersten Schritt ein niedrigerer Cut-Off-Point festgelegt als im zweiten, präziseren Schritt (Schneider u. Wagemann 2006, S. 770). Die Analyse wird gleichsam unterspezifiziert. In unserer Analyse haben wir daher für den ersten Schritt einen Cut-Off-Point von 0,75 und für den zweiten Schritt von 0,8 gewählt. 
Tabelle 6: Prüfung der politischen Instrumente auf notwendige Bedingung

\begin{tabular}{|lll|}
\hline Bedingung & Konsistenz & Abdeckung \\
\hline eco & 0,66 & 0,70 \\
norm & 0,86 & 0,70 \\
info & 0,87 & 0,70 \\
ag & 0,79 & 0,74 \\
\hline
\end{tabular}

Die Analyse ergibt, dass keine der Bedingungen notwendig für das Auftreten des Outcomes ist, denn dafür müssten die Konsistenzwerte höher sein. ${ }^{14}$

In der folgenden Analyse werden nun alle nahen Faktoren hinsichtlich hinreichender Bedingungen untersucht, unter gleichzeitiger Berücksichtigung der Wirtschaftsleistung. Die Analyse der fünf Bedingungen führt zu einer Wahrheitstafel mit 32 möglichen Konfigurationen, von denen 13 durch empirische Fälle abgedeckt sind. Begrenzte empirische Vielfalt, also der Umstand, dass - wie in unserem Fall - für einige der logisch möglichen Konfigurationen kausaler Bedingungen keine empirischen Fälle vorliegen, ist ein gängiges Problem sozialwissenschaftlicher Forschung (Ragin 1987, S. 104-113), so auch in QCA. QCA bietet jedoch die Möglichkeit eines bewussten Umgangs mit diesem Phänomen (Schneider u. Wagemann 2007 S. 106). Die QCA-Software liefert drei verschiedene Lösungsterme, die sich darin unterscheiden, welche Annahmen über die logischen Rudimente, also jene Zeilen einer Wahrheitstafel, für die keine empirische Information vorliegt, getroffen werden. Der konservative Ansatz (complex solution) macht keine Annahmen über die logischen Rudimente: Alle Konfigurationen, für die keine empirischen Fälle vorliegen, werden aus der Analyse des Outcomes ausgeschlossen. Das sparsamste Ergebnis (most parsimonious solution) dagegen beruht auf (nicht direkt kontrollierbaren) vereinfachenden Annahmen mithilfe eines Computeralgorithmus (Schneider u. Wagemann 2007, S. 106-107). Eine dritte Lösung (intermediate solution) bezieht sich auf „einfache vereinfachende Annahmen“ (Schneider u. Wagemann 2007, S. 159). Im Gegensatz zur most parsimonious solution vereinfacht diese Lösung die complex solution nicht durch Computersimulationen, sondern durch theoretische Überlegungen. So bleibt die Lösung oftmals zwar komplexer als die most parsimonious solution, verzichtet aber auf unplausible Annahmen. Dabei können für einzelne oder - wie in unserem Fall - alle Bedingungen Gedankenexperimente durchgeführt werden, wonach hohe Fuzzy-Werte in den Bedingungen tendenziell zu hohen Fuzzy-Werten im Outcome führen. Dazu müssen - wie in unserem Fall - hypothesenunterstützende Ausprägungen der Bedingungen hohe Fuzzy-Werte erhalten. ${ }^{15}$ Keiner der drei Lösungsterme wi-

14 Für notwendige Bedingungen werden generell höhere Konsistenzwerte gefordert als für hinreichende Bedingungen. In der einschlägigen Literatur wird ein Wert von mindestens 0,9 empfohlen (Schneider u. Wagemann 2007, S. 213).

15 Dabei bestehen vor allem zwei Probleme: Zum einen werden gleichsam Teile der Hypothesen als wahr vorausgesetzt, um dieselben Hypothesen auf ihren Wahrheitsgehalt überprüfen zu können. Zum anderen geht diese Denkweise implizit davon aus, dass Bedingungen alleine und nicht in Kombinationen wirken, was aber konträr zum Grundsatz der conjunctural causation in QCA ist. 
derspricht der empirisch vorhandenen Information - lediglich diejenigen theoretisch möglichen Kombinationen, für die keine empirischen Fälle vorliegen, werden unterschiedlich behandelt. ${ }^{16}$ Die drei so erzielten Lösungsformeln werden in Tabelle 7 dargestellt. Als Cut-Off-Point wurde 0,8 verwendet (siehe Fußnote 13).

\section{Tabelle 7: Ergebnis zweiter Analyseschritt}

\begin{tabular}{|l|}
\hline Parsimonious solution \\
\hline bip $\rightarrow$ elco \\
\hline Abdeckung: 0,84; Konsistenz: 0,76 \\
\hline
\end{tabular}

\begin{tabular}{|l|}
\hline Intermediate solution \\
\hline bip*ag*norm + bip*ag*info + bip*ag*eco $\rightarrow$ elco \\
\hline Abdeckung: 0,$75 ;$ Konsistenz: 0,83 \\
\hline
\end{tabular}

\begin{tabular}{|l|}
\hline Complex solution \\
\hline bip*ag*norm + bip*ag*eco* $\sim$ info + bip*ag* $\sim$ eco* info $\rightarrow$ elco \\
\hline Abdeckung: 0,$75 ;$ Konsistenz: 0,83 \\
\hline
\end{tabular}

\footnotetext{
* steht für logisches und

+ steht für logisches oder

$\sim$ bezeichnet Nichtvorliegen einer Bedingung
}

In den dargestellten Lösungstermen stellt jeder der durch ein Pluszeichen (logisches oder) getrennten Ausdrücke einen äquifinalen Pfad dar, der für sich genommen schon eine hinreichende Bedingung für das Auftreten des Outcomes ist. Es fällt auf, dass im ersten Lösungsterm trotz Berücksichtigung der nahen Faktoren weiterhin lediglich die Wirtschaftsleistung als Einflussfaktor auftritt. Werden also mithilfe des Computeralgorithmus vereinfachende Annahmen über die Wirkungsweise der logischen Rudimente getroffen, wird die Bedeutung der Wirtschaftsleistung hinsichtlich einer Reduktion des Stromverbrauchs privater Haushalte nochmals bestätigt. Die empirische Evidenz lässt also - bei entsprechenden vereinfachenden (aber nicht notwendigerweise theoriegestützten) Annahmen auch den Schluss zu, dass politische Maßnahmen keine deutliche Wirkung zeigen; allein ein niedriges Wirtschaftswachstum könnte eine hinreichende Bedingung für das Outcome sein. Allerdings liegt in unserer Analyse eine relativ große Anzahl logischer Rudimente vor. Bei der Interpretation des sparsamsten Ergebnisses ist also Vorsicht geboten. Dies ist lediglich die einfachste mögliche Lösung, die nicht

16 Mengentheoretisch gesprochen stellt jede intermediäre Lösung eine Teilmenge der sparsamsten Lösung dar, während der komplexe Lösungsterm wiederum eine Teilmenge aller anderen Lösungen ist. 
im Widerspruch zu den empirischen Daten steht. Sie sollte eher als Hinweis auf den hohen Einfluss der Wirtschaftsleistung angesehen werden, denn wie die beiden anderen Lösungen zeigen, kann - bei einem weniger automatisierten und mehr theoriegeleiteten Umgang mit logischen Rudimenten - politischen Instrumenten durchaus ein Einfluss zugeschrieben werden. Der starke Einfluss der Wirtschaftsleistung wird aber dennoch auch in den beiden anderen Lösungen deutlich, nachdem diese Bedingung in jedem Pfad dieser Lösungen auftritt. ${ }^{17}$ Es bleibt also dabei, dass vornehmlich in denjenigen Ländern ein Rückgang des Stromverbrauchs privater Haushalte zu verzeichnen ist, in denen sich die Wirtschaftsleistung im Verlauf des Untersuchungszeitraums eher schwach entwickelt hat.

Sowohl aus der intermediate solution, als auch aus der complex solution geht zudem hervor, dass keines der politischen Instrumente allein hinreichend für die Verringerung des Stromverbrauchs ist. Politische Instrumente treten ausschließlich in Verbindung miteinander oder mit anderen Faktoren in den Lösungstermen auf. Dabei spielen Institutionen eine bedeutende Rolle. Die entsprechende Bedingung (ag) tritt in jedem der Lösungsterme auf. Darüber hinaus wird deutlich, dass auch Normen und Standards einen Einfluss auf die Reduktion des Stromverbrauchs ausüben, wenn sie in Verbindung mit Institutionen eingesetzt werden. In beiden Lösungsalternativen tritt ein Lösungspfad auf, in dem dieser Einfluss durch die Kombination bip*ag*norm sichtbar wird. Demgegenüber ist der Einfluss informativer Maßnahmen und finanzieller Anreize weniger eindeutig. Zwar sind diese Bedingungen in den Lösungstermen der intermediate solution enthalten; wie jedoch aus der complex solution ersichtlich wird, ist die Art ihres Einflusses nicht unbedingt konstant, da jeweils die eine Bedingung auch als Kompensation beim Fehlen der anderen Bedingung gesehen werden kann.

Die durchweg höheren Konsistenzwerte, die im zweiten Analyseschritt in den Lösungstermen erzielt werden, in denen politische Instrumente enthalten sind, weisen darauf hin, dass unter Berücksichtigung der nahen Faktoren das Outcome besser erklärt werden kann als im ersten Analyseschritt. Allerdings decken diese Lösungsterme auch einen geringeren Anteil des Outcomes ab, wie aus den niedrigeren Abdeckungsmaßen hervorgeht.

Zusammenfassend lässt sich sagen, dass unter Berücksichtigung des Kontextes einer schwachen Wirtschaftsleistung diejenigen Länder besonders erfolgreich bei der Senkung des privaten Stromverbrauchs waren, in denen spezifische Institutionen eingerichtet wurden, die sich der Steigerung der Energieeffizienz annehmen. In Verbindung mit diesen Institutionen haben sich zudem Normen und Standards als wirksame Instrumente im Hinblick auf eine Reduktion des privaten Stromverbrauchs erwiesen. Hinsichtlich der Effekte informativer und finanzieller Maßnahmen liefert die Analyse jedoch keine eindeutigen Ergebnisse.

In Anbetracht dieser Ergebnisse kann unsere erste Hypothese größtenteils bestätigt werden, die besagt, dass es nur dann zu deutlichen Stromeinsparungen in privaten Haushalten kommt, wenn in einem Land mehrere politische Instrumente zur Steigerung der Energieeffizienz eingesetzt werden. Zwar ist auch der Kontext

17 Obwohl dies eine rein mathematische Konsequenz ist (alle anderen Lösungen sind Teilmengen der sparsamsten Lösung), mindert dies nicht die Bedeutung des Faktors. 
einer schwachen Wirtschaftsentwicklung von großer Bedeutung, unsere Ergebnisse heben jedoch zugleich den Einfluss politischer Instrumente hervor.

Die Tatsache, dass Institutionen in Kombination mit anderen politischen Instrumenten eine zentrale Bedeutung zukommt, lässt sich anhand der spezifischen Charakteristika dieses Instrumententyps erklären. Institutionen wie Energiesparfonds oder Energieagenturen tragen dazu bei, verschiedene Hemmnistypen und -ebenen gleichzeitig zu adressieren. Sie dienen darüber hinaus der zentralen Koordination der eingesetzten, häufig sehr vielfältigen Policies und fördern somit die Stringenz der auf nationaler Ebene verfolgten Politik zur Förderung der Energieeffizienz.

Der mitunter zentrale Einfluss, der Normen und Standards zukommt, lässt sich dadurch begründen, dass Instrumente, die über die Verbraucherumgebung indirekt auf den Verbraucher einwirken, besonders effektiv sind, da sie entscheidend zur Schaffung einer förderlichen Verbraucherumgebung beitragen können.

Um der Frage nach dem zentralen Einfluss von Institutionen weiter nachzugehen, wurde in einer separaten Fallstudie, die hier nicht in vollem Umfang dargestellt werden kann, der politische Ansatz untersucht, der in Dänemark verfolgt wird (Mayer 2009). Sowohl die Ergebnisse der Analyse als auch eine umfassende Literaturanalyse weisen darauf hin, dass Dänemark überdurchschnittliche Erfolge im Bereich Energieeffizienz aufzuweisen hat, die mit einer breit angelegten politischen Intervention einhergehen.

Der dänischen Stromsparfonds soll hier exemplarisch skizziert werden, um so den als zentral identifizierten Einfluss von Institutionen empirisch zu unterstreichen. Die Arbeit des Fonds orientiert sich an einer Strategie, die auf spezifischen Grundsätzen basiert. Zum einen soll es für die Verbraucher einfach, sicher und kostengünstig sein, Energie einzusparen. Zum anderen soll auf der Angebotsseite die Marktdurchdringung energieeffizienter Geräte gefördert werden. Dabei wird eine enge Zusammenarbeit mit relevanten Herstellern und Händlern anvisiert. Zu den maßgeblichen Initiativen zählen in diesem Zusammenhang groß angelegte Informations- und Marketingkampagnen (siehe dazu auch Karbo 2001) sowie freiwillige Vereinbarungen mit den Produzenten, wenig energieeffiziente Geräte vom Markt zu nehmen, wenn sich der Fonds im Gegenzug für die Vermarktung energieeffizienterer Geräte einsetzt. Ein weiterer Schwerpunkt des Fonds liegt darin, die Substitution elektrischer Heizsysteme durch Fernwärme zu fördern. Die verschiedenen, vom Fonds ausgehandelten Rahmenvereinbarungen für den Einbau der neuen Anlagen führten dazu, dass die Kosten der Umstellung für die Verbraucher halbiert werden konnten.

\section{Fazit und Ausblick}

Ziel dieser Arbeit war es, zu untersuchen, ob und unter welchen Bedingungen politische Maßnahmen zur Steigerung von Energieeffizienz insoweit Wirkung zeigen, dass in privaten Haushalten weniger Strom verbraucht wird. Dabei stand die Frage im Vordergrund, welche politischen Instrumente den größten Einfluss haben, bzw. in welchen Kombinationen und unter welchen Bedingungen diese am wirkungsvollsten sind. 
Warum politische Intervention überhaupt erforderlich ist, erklärt sich dadurch, dass mehrere Arten von Marktversagen der Ausnutzung des vorhandenen Potenzials zur Energieeffizienzsteigerung im Wege stehen. Unsere empirische Analyse hat gezeigt, dass die sehr unterschiedlich gelagerten Hemmnisse nur mit einer spezifischen Kombination von institutionellen Arrangements, Politikinstrumenten und sozioökonomischen Rahmenbedingen überwunden werden können. Es wurde deutlich, dass unter Berücksichtigung des Kontextfaktors einer schwachen wirtschaftlichen Entwicklung spezifische politische Instrumente in unterschiedlichen Kombinationen einen Einfluss darauf haben können, dass in privaten Haushalten weniger Strom verbraucht wird. Während institutionellen Arrangements wie Energieeffizienzagenturen ein zentraler Einfluss zugeschrieben werden kann, kommt Normen und Standards in Kombination mit diesen Institutionen eine eher ergänzende Rolle zu. Informative und finanzielle Instrumente zeigen demgegenüber einen weniger eindeutigen Einfluss.

Diese Studie ist unseres Wissens nach die erste, die einen systematischen internationalen Vergleich von Energieeffizienzpolitiken mittels QCA durchführt. Die Ergebnisse der Studie liefern jedoch nur einen ersten Einblick in die Wirkungsweise politischer Maßnahmen zur Steigerung von Energieeffizienz. Weiterführende Forschung mit ergänzenden Methoden (z. B. Fallstudien und Zeitreihenanalysen) wäre wünschenswert, um noch mehr Klarheit in Bezug auf die spezifischen Wirkungsmechanismen und differentiellen Effekte der Politikinstrumente zu erlangen. Mit dem von uns verwendeten Instrumentenindex konnten wir dem Ziel einer möglichst umfassenden Vergleichbarkeit der zur Verfügung stehenden Daten bereits recht nahe kommen. Eine internationale Koordination und Harmonisierung der Evaluationsmethoden würde diesem Anliegen jedoch zusätzlich Vorschub leisten. Zukünftige Forschung könnte in dieser Hinsicht von einem Ausbau der bisher noch sehr lückenhaften Evaluation nationaler Programme zur Förderung der Energieeffizienz profitieren. Zumindest auf europäischer Ebene werden mittlerweile Anstrengungen unternommen, die in den einzelnen Mitgliedsstaaten erzielten Einsparungen durch standardisierte Evaluationsverfahren vergleichbarer zu machen (siehe dazu Wuppertal Institut 2009).

\section{Literatur}

Abrahamse, Wokje, Linda Steg, Charles Vlek, und Talib Rothengatter. 2005. A Review of Intervention Studies Aimed at Household Energy Conservation. Journal of Environmental Psychology 25:273-291.

Banerjee, Abhijit, und Barry D. Solomon. 2003. Eco-Labeling for Energy Efficiency and Sustainability: A Meta-Evaluation of US Programs. Energy Policy 31:109-123.

Befani, Barbara, Simone Ledermann, und Fritz Sager. 2007. Realistic Evaluation and QCA: Conceptual Parallels and an Empirical Application. Evaluation 13:171-192.

Berry, David. 2008. The Impact of Energy Efficiency Programs on the Growth of Electricity Sales. Energy Policy 36: 3620-25.

Blatter, Joachim, Frank Janning, und Claudius Wagemann. 2007. Qualitative Politikanalyse: eine Einführung in Forschungsansätze und Methoden. Wiesbaden: VS Verlag für Sozialwissenschaften. 
Blumstein, Carl, Betsy Krieg, Lee Schipper, und Carl York. 1980. Overcoming Social and Institutional Barriers to Energy Conservation. Energy 5:355-372.

Braun, Dietmar, und Olivier Giraud. 2008. Politikinstrumente im Kontext von Staat, Markt und Governance. In Lehrbuch der Politikfeldanalyse 2.0, Hrsg. Klaus Schubert und Nils C. Bandelow, 159-186. München: Oldenbourg.

Brown, Marilyn. 2001. Market Failures and Barriers as a Basis for Clean Energy Policies. Energy Policy 29:1197-1207.

Duscha, Markus. 2008. Bausteine für eine kohärente Strategie zur Förderung der Stromeffizienz in den privaten Haushalten. In Strom sparen im Haushalt. Trends, Einsparpotenziale und neue Instrumente für eine nachhaltige Energiewirtschaft, Hrsg. Corinna Fischer, 145-157. München: oekom.

Geller, Howard, Philipp Harrington, Arthur H. Rosenfeld, Satoshi Tanishima, und Fridtjof Unander. 2006. Policies for Increasing Energy Efficiency: Thirty Years of Experience in the OECD Countries. Energy Policy 34:556-573.

Gillingham, Kenneth, Richard G. Newell, und Karen Palmer. 2006. Energy Efficiency Policies: A Retrospective Examination. Annual Review of Environment and Resources 31:161-192.

Gillingham, Kenneth, Richard G. Newell, und Karen Palmer. 2009. Energy Efficiency Economics and Policy. Annual Review of Resource Economics 1:597-620.

Goldstein, Daniel G., und Gerd Gigerenzer. 2002. Models of Ecological Rationality: The Recognition Heuristic. Psychological Review 109:75-90.

Görlitz, Axel, und Hans-Peter Burth. 1998. Politische Steuerung. Ein Studienbuch. Opladen: Leske + Budrich.

Harmelink, Mirjam, Lars Nilsson, und Robert Harmsen. 2008. Theory-Based Policy Evaluation of 20 Energy Efficiency Instruments. Energy Efficiency 1:131-148.

Henley, Andrew, und John Peirson. 1998. Residential Energy Demand and the Interaction of Price and Temperature: British Experimental Evidence. Energy Economics 20:157171.

Henryson, Jessica, Teresa Håkansson, und Jurek Pyrko. 2000. Energy Efficiency in Buildings Through Information - Swedish Perspective. Energy Policy 28:169-180.

Holzinger, Katharina. 2009. Vom ungeliebten Störenfried zum akzeptierten Paradigma? Zum Stand der (Neuen) Politischen Ökonomie in Deutschland. Politische Vierteljabresschrift 50:539-576.

IEA. 2003. Cool Appliances. Policy Strategies for Energy Efficient Homes. Paris: OECD/ International Energy Agency.

IEA. 2004. Oil Crises and Climate Challenges. 30 Years of Energy Use in IEA Countries. Paris: OECD/ International Energy Agency.

IEA. 2007. Mind the gap. Quantifying Principal-Agent Problems in Energy Efficiency. Paris: OECD/ International Energy Agency.

IEA. 2009. Gadgets and Gigawatts. Policies for Energy Efficient Electronics. Paris: OECD/ International Energy Agency.

Jaffe, Adam, und Robert N. Stavins. 1994. The Energy-Efficiency Gap. What Does it Mean? Energy policy 22:804-810.

Kahneman, Daniel, und Amor Tversky. 2000. Choices, Values, and Frames. Cambridge: Cambridge University Press. 
Karbo, Peter. 2001. The Danish Electricity Saving Trust: The Campaign in Autumn 1999 - A Review of Experience. In Energy Efficiency in Household Appliances and Lighting, Hrsg. Paolo Bertoldi, Andrea Ricci und Anibal de Almeida, 796-805. Heidelberg: Springer.

Kraft, John, und Arthur Kraft. 1978. On the Relationship Between Energy and GNP. Journal of Energy and Development 3:401-403.

Mayer, Ines. 2009. Energieeffizienz in privaten Haushalten im internationalen Vergleich. Eine Policy-Wirkungsanalyse mit QCA. Konstanz: Masterarbeit an der Universität Konstanz. http://kops.ub.uni-konstanz.de/volltexte/2010/11349/pdf/Dipl_Mayer_Energieeffizienz.pdf.

McFadden, Daniel. 1999. Rationality for Economists? Journal of Risk and Uncertainty 19:73-105.

Mackie, John L. 1974. The Cement of the Universe. A Study of Causation. Oxford: Clarendon Press.

Metcalf, Gilbert E. 2008. An Empirical Analysis of Energy Intensity and its Determinants at the State Level. The Energy Journal 29:1-26.

Nadel, Steven. 2002. Appliance and Equipment Efficiency Standards. Annual Review of Energy and the Environment 27:159-192.

Nordqvist, Joakim. 2006. Evaluation of Japan's Top Runner Programme. Within the Framework of the AID-EE Project. http://www.aid-ee.org/documents/018TopRunnerJapan.PDF. Zugegriffen 17.06.2010.

OECD. 2008a. Household Behaviour and the Environment. Reviewing the Evidence. Paris: Organisation for Economic Co-operation and Development.

OECD. 2008b. OECD Environmental Outlook to 2030. Paris: Organisation for Economic Co-operation and Development.

Ragin, Charles C. 1987. The Comparative Method. Moving Beyond Qualitative and Quantitative Strategies. Berkeley: University of California Press.

Ragin, Charles C. 2000. Fuzzy-Set Social Science. Chicago: University of Chicago Press.

Ragin, Charles C. 2008. Redesigning Social Inquiry. Fuzzy Sets and Beyond. Chicago: University of Chicago Press.

Reisch, Lucia A., und Andreas Oehler. 2009. Behavioral Economics: Eine neue Grundlage für die Verbraucherpolitik? Vierteljahrshefte zur Wirtschaftsforschung 78:30-43.

Reiss, Peter C., und Matthew W. White. 2008. What Changes Energy Consumption? Prices and Public Pressures. The RAND Journal of Economics 39:636-663.

Rihoux, Benoît, und Heike M. Grimm. 2006. Innovative Comparative Methods for Policy Analysis. Beyond the Quantitative-Qualitative Divide. New York: Springer.

Scharpf, Fritz W. 2006. Interaktionsformen. Akteurzentrierter Institutionalismus in der Politikforschung. Wiesbaden: VS Verlag für Sozialwissenschaften.

Schipper, Lee, und Michael Grubb. 2000. On the Rebound? Feedback Between Energy Intensities and Energy Uses in IEA Countries. Energy Policy 28:367-388.

Schneider, Carsten Q., und Claudius Wagemann. 2006. Reducing Complexity in Qualitative Comparative Analysis (QCA): Remote and Proximate Factors and the Consolidation of Democracy. European Journal of Political Research 45:751-786.

Schneider, Carsten Q., und Claudius Wagemann. 2007. Qualitative Comparative Analysis und Fuzzy Sets. Ein Lehrbuch für Anwender und jene, die es werden wollen. Opladen: Barbara Budrich. 
Schneider, Carsten Q., und Claudius Wagemann. 2010. Standards of Good Practice in Qualitative Comparative Analysis (QCA) and Fuzzy-Sets. Comparative Sociology 9:397-418.

Simon, Herbert A. 1957. Models of Man. London: Wiley.

Sorrell, Steve. 2004. Understanding Barriers to Energy Efficiency. In The Economics of Energy Efficiency. Barriers to Cost-Effective Investment, Hrsg. Steve Sorrell, Eoin O’Malley, Joachim Schleich und Sue Scott, 25-94. Cheltenham: Edgar Elgar.

Sorrell, Steve. 2007. The Rebound Effect: An Assessment of the Evidence for EconomyWide Energy Savings from Improved Energy Efficiency. London: UK Energy Research Center.

Soytas, Ugur, und Ramazan Sari. 2003. Energy Consumption and GDP: Causality Relationship in G-7 Countries and Emerging Markets. Energy Economics 25:33-37.

Tenbücken, Marc, und Janina Thiem. 2004. Facettentheoretische Verfahren in der Politikwissenschaft - eine Einführung. In Der Staat auf dem Rückzug. Die Privatisierung öffentlicher Infrastrukturen, Hrsg. Volker Schneider und Marc Tenbücken, 317-326. Frankfurt a. M.: Campus Verlag.

Ürge-Vorsatz, Diana, Sonja Koeppel, und Sebastian Mirasgedis. 2007. Appraisal of Policy Instruments for Reducing Buildings' CO2 Emissions. Building Research and Information 35:458-477.

Vedung, Evert. 1998. Policy Instruments: Typologies and Theories. In Carrots, Sticks and Sermons. Policy Instruments and their Evaluation, Hrsg. Marie-Louise BemelmansVidec, Ray C. Rist und Evert Vedung, 21-58. New Brunswick: Transaction Publishers.

Verkuilen, Jay. 2005. Assigning Membership in a Fuzzy Set Analysis. Sociological Methods \& Research 33:462-496.

Wagemann, Claudius. 2008. Qualitative Comparative Analysis und Policy-Forschung. In Die Zukunft der Policy-Forschung, Hrsg. Frank Janning und Katrin Toens, 242-258. Wiesbaden: VS Verlag für Sozialwissenschaften.

Windhoff-Héritier, Adrienne. 1987. Policy-Analyse. Eine Einführung. Frankfurt a. M.: Campus Verlag.

World Energy Council. 2008. Energy Efficiency Policies around the World: Review and Evaluation. London: World Energy Council.

Wuppertal Institut. 2009. Measuring and Reporting Energy Savings for the Energy Services Directive - How It Can Be Done. Results and Recommendations from the EMEEES Project. http:/www.evaluate-energy-savings.eu/emeees/en/publications/reports/EMEEES_ Final_Report.pdf. Zugegriffen: 17.06.2010.

Yuan, Chaoqing, Sifeng Liu, Zhigeng Fang, und Junlong Wu. 2009. Research on the Energy-Saving Effect of Energy Policies in China: 1982-2006. Energy Policy 37:2475-2480. 


\section{Autorenangaben:}

Ines Mayer, M.A., Europäisches Institut für Energieforschung (EIFER), Emmy-Noether-Str. 11, 76131 Karlsruhe, mayer@eifer.org

[Korrespondenzautorin]

Prof. Dr. Volker Schneider,

Universität Konstanz, Fachbereich für Politik- und Verwaltungswissenschaft, Fach D 81, 78457 Konstanz,

volker.schneider@uni-konstanz.de

Dr. Claudius Wagemann,

Istituto italiano di scienze umane (SUM), Palazzo Strozzi, Piazza degli Strozzi, I-50123 Florenz, Italien, claudius.wagemann@sumitalia.it 